\title{
Effectiveness and Safety of Rivaroxaban versus Warfarin Among Nonvalvular Atrial Fibrillation Patients with Obesity and Polypharmacy
}

\author{
Mark J. Alberts ${ }^{1}$. Jinghua He ${ }^{2} \cdot$ Akshay Kharat $^{2} \cdot$ Veronica Ashton $^{2}$
}

Accepted: 13 December 2021 / Published online: 29 January 2022

(c) The Author(s) 2022

\begin{abstract}
Background Current evidence suggests that rivaroxaban may be well tolerated and effective in patients with nonvalvular atrial fibrillation (NVAF) and obesity; however, there is limited evidence on the impact of polypharmacy in this population. This study evaluated real-world clinical outcomes with rivaroxaban versus warfarin in patients with NVAF and obesity according to the number of concurrent medications.

Methods This retrospective cohort study identified patients with one or more pharmacy claim for rivaroxaban or warfarin from two large claims databases. Patients were required to have an atrial fibrillation diagnosis, body mass index $\geq 30 \mathrm{~kg} / \mathrm{m}^{2}$ and the presence of polypharmacy (1-4,5-9, or $\geq 10$ concurrent medications). Outcomes of stroke, systemic embolism, and major bleeding were compared between the rivaroxaban and warfarin cohorts after propensity score matching (PSM). Results A total of 95,875 patients were identified with one or more claim for either rivaroxaban or warfarin. After PSM, patient characteristics were balanced between cohorts $(n=21,547$ in each cohort). The overall composite risk of stroke and systemic embolism was significantly lower in the rivaroxaban cohort compared with the warfarin cohort (hazard ratio [HR] $0.77,95 \%$ confidence interval $[\mathrm{CI}] 0.70-0.84 ; p<0.001$ ). The risks of ischemic stroke, hemorrhagic stroke, and systemic embolism separately were also significantly reduced with rivaroxaban. Major bleeding risk was similar between cohorts (HR $0.93,95 \%$ CI $0.81-1.06 ; p=0.2842$ ), and results were consistent across the three polypharmacy groups.
\end{abstract}

Conclusions In this real-world study of NVAF patients with obesity, rivaroxaban was associated with lower risks of stroke and systemic embolism and similar risk of major bleeding versus warfarin across polypharmacy categories.

\section{Key Points}

Among nonvalvular atrial fibrillation patients with obesity and polypharmacy, the risk of stroke/systemic embolism was significantly lower with rivaroxaban versus warfarin, with similar major bleeding risk.

The risks of ischemic stroke, hemorrhagic stroke, and systemic embolism separately were significantly lower with rivaroxaban versus warfarin.

Results were consistent across the three polypharmacy groups.

Mark J. Alberts

mark.alberts@hhchealth.org

1 Hartford HealthCare, 85 Seymour St \#815, Hartford, CT 06106, USA

2 Janssen Scientific Affairs, LLC, Titusville, NJ, USA

\section{Introduction}

Atrial fibrillation (AF) is a common cardiac arrhythmia that predominately affects older adults (mean age $\geq 70$ years) [1]. The risk of stroke is increased fivefold in patients with nonvalvular AF (NVAF), and strokes are more likely to be severe in NVAF patients [2]. Obesity is a worsening public health crisis associated with serious health consequences, challenges for healthcare providers, and substantial economic burden [3-5]. The prevalence of adult obesity in the US was estimated at $42 \%$ in 2017-2018 and may approach $50 \%$ by 2030 [6-8]. In addition to other health problems, obesity is associated with a higher risk of developing NVAF $[9,10]$ and thrombotic events $[1,11$, 12]. In obese patients who develop NVAF, the condition is both severe and more persistent [13]. Obese patients are also likely to have comorbid conditions, including NVAF, that require concomitant use of multiple medications (i.e., polypharmacy) [14, 15]. Polypharmacy is a concern for obese patients with NVAF as additional medications may 
lead to poor treatment adherence or drug-drug interactions that reduce efficacy, thereby increasing the risk of adverse clinical outcomes and mortality $[16,17]$.

Direct oral anticoagulants (DOACs) are recommended as the standard of care to treat and prevent thromboembolic events in patients with NVAF [18]. In 2016, the International Society of Thrombosis and Haemostasis recommended standard dosing of DOACs in patients with a body mass index (BMI) of $40 \mathrm{~kg} / \mathrm{m}^{2}$ or less, but did not recommend use of DOACs in morbidly obese patients (BMI $>40 \mathrm{~kg} / \mathrm{m}^{2}$ ) due to limited clinical evidence in this population [12]. Subsequently, a number of studies have evaluated the use of DOACs in patients with morbid obesity (defined as body weight $>120 \mathrm{~kg}$ or BMI $>40 \mathrm{~kg} / \mathrm{m}^{2}$ ), and two systematic reviews suggest that the benefit-risk profile of DOACs, particularly rivaroxaban and apixaban, are preserved in this population $[19,20]$. Rivaroxaban, an oral direct factor Xa inhibitor, was approved for the prevention of stroke and systemic embolism in patients with NVAF in November 2011 based on data from the ROCKET-AF trial [21, 22]. Of note, more than two-thirds of patients in the ROCKET-AF trial were on five or more concurrent medications in addition to their anticoagulant therapy; a subgroup analysis of the trial found no difference in the risk of outcomes between rivaroxaban and warfarin, except for bleeding, based on the number of concurrent medications [16]. Several real-world studies have also examined the effectiveness of rivaroxaban in NVAF patients, separately in patients with obesity [23-27] and patients with polypharmacy [28, 29]. In general, rivaroxaban treatment was associated with a reduced risk of stroke-related outcomes compared with warfarin, with similar effects on bleeding in these separate populations.

Due to the high likelihood of the presence of both obesity and polypharmacy in NVAF patients, this study evaluated the real-world effectiveness and safety of rivaroxaban compared with warfarin in a population of NVAF patients with both obesity and polypharmacy. The primary objectives of the study were to compare the risk of stroke (ischemic or hemorrhagic) or systemic embolism and the risk of major bleeding overall among NVAF patients with obesity and one or more concurrent medications, and by polypharmacy groups (i.e., 1-4, 5-9, and $\geq 10$ concurrent medications). The secondary objective was to evaluate and compare the persistence and adherence to the index treatment.

\section{Methods}

\subsection{Data Sources}

Two large claims databases were combined for these analyses: IBM MarketScan Commercial Claims and Encounters (CCAE) and IBM MarketScan Medicare Supplemental
(MDCR). CCAE is a fully adjudicated paid medical and pharmacy insurance claims database of approximately 138 million unique de-identified persons, including active employees, early retirees, COBRA continuers, and their dependents insured by employer-sponsored plans. CCAE includes inpatient admission records, outpatient services, prescription drugs, enrollment statuses, and costs of medical services and drugs. MDCR is an administrative health claims database for Medicare-eligible active and retired employees and their Medicare-eligible dependents from employer-sponsored supplemental plans. MDCR captures person-specific clinical utilization, cost, and enrollment across inpatient, outpatient, prescription drug, and carveout services.

\subsection{Study Design}

This retrospective cohort study identified NVAF patients with obesity and polypharmacy initiating rivaroxaban or warfarin treatment between 1 December 2011 and 1 March 2020 (Fig. 1).

\subsection{Patients}

Patients newly treated with rivaroxaban or warfarin were identified from the database based on having one or more pharmacy claims for rivaroxaban or warfarin during the identification period, with the first claim defined as the index date; $\geq 12$ months of continuous medical and pharmacy benefit enrollment prior to and on the index date; and one or more medical claims with a diagnosis of AF (International Classification of Diseases, Ninth Revision, Clinical Modification [ICD-9-CM] code 427.31 and International Classification of Diseases, Tenth Revision, Clinical Modification [ICD-10-CM] codes I48.0\%-I48.2\%, I $48.91 \%$ ) during the 12-month period prior to or on the index date. Patients were also required to be $\geq 18$ years of age and have obesity (defined as BMI $\geq 30 \mathrm{~kg} / \mathrm{m}^{2}$ ), which was determined based on a proprietary validated BMI interpolation algorithm [30]. The algorithm adopted a novel automated weighted prediction approach (Super Learner algorithm), which leveraged the predictions from four different machine learning algorithms (Catboost, random forest, least absolute shrinkage and selection operator [LASSO] regression, and artificial neural networks) through logistic regression. Features included diagnoses, procedures, and medication use during the 12-month baseline period and patient demographics.

The algorithm was trained in the Optum Clinformatics Date of Death (Optum DOD) database and validated both internally in the Optum DOD and externally in the IBM CCAE database based on assessments of area under the receiver operating characteristic curve (ROC AUC), $F 1$ 


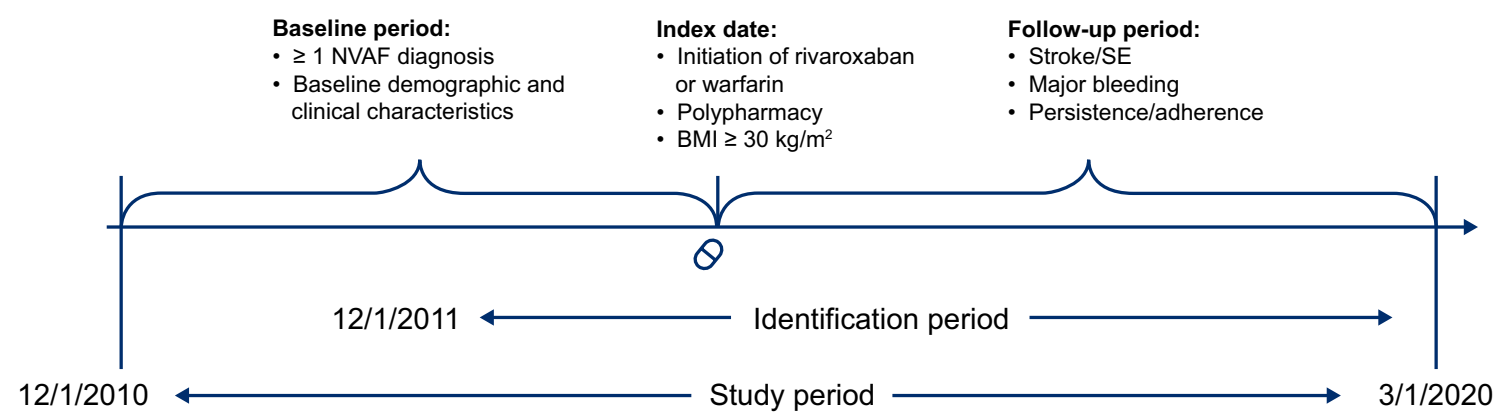

Fig. 1 Study design. The identification period was from 1 December 2011 to 1 March 2020. The index event was the first pharmacy dispensing for rivaroxaban or warfarin during the identification period, while the index date was the first claim date for the initiation of rivaroxaban or warfarin. The baseline period was a 12 -month period with

score, accuracy, negative predictive value, specificity, positive predictive value or precision, and sensitivity or recall. The predictive models to determine BMI classifications using claims data were based on the patient's baseline profile [30]. If patients had a baseline BMI diagnosis code, then model 1 was applied; however, for patients without a BMI diagnosis code, model 2 was applied. Model 1 had a ROC AUC of approximately $88 \%$ for predicting BMI classifications of $\geq 30, \geq 35$, and $\geq 40 \mathrm{~kg} / \mathrm{m}^{2}$, and accuracy and specificity ranged from 88 to $93 \%$ and 92 to $95 \%$, respectively. For model 2, ROC AUC was 73\%, and accuracy and specificity ranged from 74 to $80 \%$ and 72 to $86 \%$, respectively.

The presence of polypharmacy was based on the number of concurrent medications used in addition to rivaroxaban or warfarin on the index date. Concurrent use was determined by the date of the prescription, days' supply, and a grace period of 14 days. Different pharmacy prescriptions were identified by unique generic product identifier codes at an 8-digit level (or 10-digit level for fixed-dose combinations). Polypharmacy status was categorized as $1-4,5-9$, and $\geq 10$ concurrent medications on the index date [16].

Patients were excluded if they had a diagnosis code for stroke, systemic embolism, or major bleeding within 30 days prior to the index date; pharmacy claims for two or more oral anticoagulant medications on the index date; or one or more pharmacy claims for an oral anticoagulant at any time prior to the index date. Patients with evidence of another indication for anticoagulation (e.g., acute venous thromboembolism, prophylaxis after hip/knee replacement surgery) during the baseline period were excluded. Patients with a diagnosis code for mitral stenosis or a diagnosis or procedure code for a mechanical heart valve procedure at any time prior to the index date were excluded. Patients with no pharmacy claims for other concurrent medications in addition to rivaroxaban or warfarin were also excluded. continuous health plan enrollment prior to the index date. Criteria for inclusion in the 12-month baseline period could be met at any time during this period. $B M I$ body mass index, $N V A F$ nonvalvular atrial fibrillation, $S E$ systemic embolism

\subsection{Outcomes}

Stroke and systemic embolism were defined as a hospitalization or emergency room visit with a primary diagnosis of stroke (ischemic or hemorrhagic) or systemic embolism (ischemic stroke: ICD-9-CM: 433.01, 433.11, 433.21, 433.31, 433.81, 433.91, 434.01, 434.11, 434.91, 436.\%; ICD-10-CM: I63.\%; hemorrhagic stroke: ICD-9-CM: 430.\%, 431.\%, 432.9; ICD-10-CM: I60.\%, I61.\%, I62.\%; systemic embolism: ICD-9-CM: 444.01, 444.09, 444.1, 444.21, 444.22, 444.81, 444.89, 444.9; ICD-10-CM: I74.\%). Stroke and systemic embolism were analyzed using an intent-totreat approach (i.e., the earliest of the first stroke/systemic embolism event, health plan disenrollment, or latest data availability) and were first measured as a composite endpoint and then measured as separate components.

Major bleeding was identified during the follow-up period using a validated claims-based algorithm developed by Cunningham et al. [31]. Hemorrhagic stroke is included in the definition of a major bleeding event. Major bleeding was analyzed using an as-treated approach (i.e., the earliest of the first major bleeding event, index treatment discontinuation, switching to or adding another anticoagulant, health plan disenrollment, or latest data availability).

Persistence and adherence to the index treatment were defined as the time-to-first discontinuation and the proportion of days covered (PDC) during the follow-up period, respectively. Discontinuation was defined as no subsequent dispensing of the index medication prior to the end of the 60-day maximum permissible gap of a dispensing (i.e., days elapsed between the last date of days' supply of a dispensing and the subsequent dispensing of the same medication). PDC is the ratio of the number of days covered by the index medication prescription dispensed during the follow-up period divided by the number of days of the entire followup period. PDC values range from 0.01 to 1.00 , with higher values suggesting higher compliance. 


\subsection{Statistical Analysis}

Propensity score matching (PSM) was used to reduce potential bias and create more comparable cohorts of rivaroxaban and warfarin users based on baseline characteristics. A logistic regression model was used to predict the propensity of receiving rivaroxaban with consideration of the following potential confounders: age; sex; geographic region; health plan type; insurance type; index year; polypharmacy status (1-4, 5-9, and $\geq 10$ ); BMI status (BMI 30.0-34.9 kg/m², $35.0-39.9 \mathrm{~kg} / \mathrm{m}^{2}$, and $\geq 40 \mathrm{~kg} / \mathrm{m}^{2}$ ); Quan-Charlson comorbidity index (QCI) [32]; $\mathrm{CHA}_{2} \mathrm{DS}_{2}$-VASc score [33]; HASBLED score [34]; cancer diagnosis, gastric bypass surgery, and cardiovascular procedures during the 12-month baseline period; baseline occurrence or use of non-oral anticoagulants, antihyperlipidemics, antihypertensives, or antiplatelet agents [31]; and all-cause baseline healthcare resource utilization and costs. Rivaroxaban users were matched 1:1 with warfarin users using PSM without replacement using calipers of width equal to $20 \%$ of the standard deviation (SD) of the logit of the propensity score.

Demographics and baseline characteristics for each treatment cohort were summarized with descriptive statistics; differences in baseline characteristics were assessed using standardized differences, with a difference of $<10 \%$ considered a negligible imbalance. The outcomes of stroke, systemic embolism, and major bleeding were compared between propensity score-matched treatment cohorts; these outcomes were not compared in the unmatched groups due to expected bias from confounding factors that would prevent our ability to draw conclusions about the data. Stroke and bleeding outcomes were analyzed as time-to-event; if no events are observed, patients were censored at the end of the follow-up.

Cox proportional hazards models were used to calculate hazard ratios (HRs), 95\% confidence intervals (CIs), and $p$-values [35]; Kaplan-Meier curves were also generated. Comparative analyses were performed overall, by polypharmacy groups (1-4, 5-9, and $\geq 10$ concurrent medications) and BMI cat-

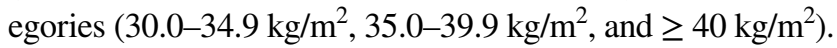
Sensitivity analyses were conducted to evaluate the robustness of the findings: (1) intent-to-treat analyses were repeated using an as-treated approach, and (2) obesity status was identified by using obesity ICD-9-CM and ICD-10-CM diagnosis codes during the baseline period rather than using the BMI interpolation algorithm (Electronic Supplementary Table 1).

\section{Results}

\subsection{Patient Characteristics}

Of the 95,875 patients who met all eligibility criteria, 33,191 patients were treated with rivaroxaban and 62,684 patients were treated with warfarin (Fig. 2). Prior to matching, rivaroxaban patients were younger than warfarin patients and had lower QCI, $\mathrm{CHA}_{2} \mathrm{DS}_{2}$-VASc, and HAS-BLED scores. After 1:1 PSM, 21,547 matched pairs were generated that were balanced (i.e., standardized differences $<10 \%$ ) between cohorts for demographics, baseline clinical characteristics, and comorbid conditions (Table 1).

\subsection{Outcomes}

In the overall population, the composite risk of stroke and systemic embolism was significantly lower in the rivaroxaban cohort compared with the warfarin cohort over a mean follow-up time of 25 months ( $4.3 \%$ vs. $5.6 \%$; HR $0.77,95 \%$ CI $0.70-0.84 ; p<0.001$ ) (Figs. 3 and 4). Rivaroxaban was also associated with a significantly lower risk of ischemic stroke, hemorrhagic stroke, and systemic embolism versus warfarin. The risk of major bleeding was not significantly different in the rivaroxaban cohort versus warfarin cohort over a mean followup time of 12 and 11 months, respectively (2.0\% vs. $2.0 \%$; HR 0.93, 95\% CI 0.81-1.06; $p=0.2842$ ) (Figs. 3 and 4).

Consistent with the overall population, the risk of the composite outcome of stroke and systemic embolism and ischemic stroke alone was lower with rivaroxaban compared with warfarin across polypharmacy subgroups (Fig. 3). No difference in the risk of major bleeding was seen across polypharmacy groups with rivaroxaban compared with warfarin.

In a subgroup analysis by baseline BMI category, the risk of the composite outcome of stroke and systemic embolism, ischemic stroke, hemorrhagic stroke, and systemic embolism was lower in the rivaroxaban cohort compared with the warfarin cohort regardless of baseline BMI category (30.0-34.9 $\mathrm{kg} / \mathrm{m}^{2}, 35.0-39.9 \mathrm{~kg} / \mathrm{m}^{2}$, and $\geq 40 \mathrm{~kg} / \mathrm{m}^{2}$ ) [Fig. 5]. The risk of major bleeding was not different between treatment cohorts across BMI categories, although the risk was borderline significantly lower in the rivaroxaban cohort versus the warfarin cohort for patients whose BMI was $\geq 40 \mathrm{~kg} / \mathrm{m}^{2}$.

Results from a sensitivity analysis using an as-treated approach for the effectiveness outcomes were consistent with the intent-to-treat analyses, with significantly lower risks of the composite outcome of stroke/systemic embolism, ischemic stroke, hemorrhagic stroke, and systemic embolism in the rivaroxaban cohort compared with the warfarin cohort (Electronic Supplementary Table 2). Sensitivity analyses based on the classification of BMI using diagnosis codes were generally consistent with findings from the primary analysis (Electronic Supplementary Tables 3 and 4).

\subsection{Persistence and Adherence}

The mean time to index treatment discontinuation was 12.4 months and 11.3 months for the rivaroxaban and warfarin 
Fig. 2 Patient attrition. $A F$ atrial fibrillation, $B M I$ body mass index, $D V T$ deep vein thrombosis, $E R$ emergency room, $G P I$ generic product identifier, HCPCS healthcare common procedure coding system, ICD-9-CM International Classification of Diseases, Ninth Revision, Clinical Modification, ICD-10-CM International Classification of Diseases, Tenth Revision, Clinical Modification, $P E$ pulmonary embolism

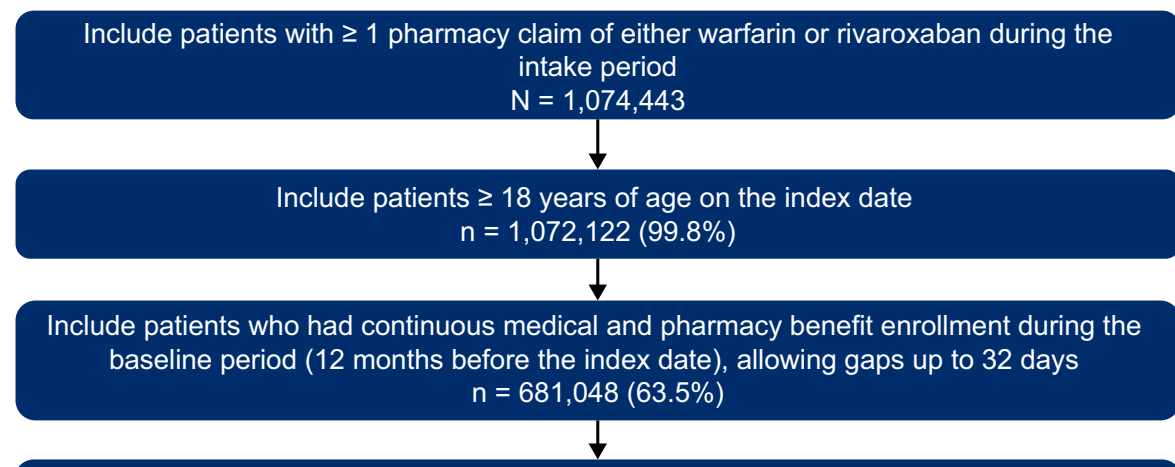

Include patients with $\geq 1$ medical claim with ICD-9-CM or ICD-10-CM diagnosis code(s) for AF during the baseline period and/or on the index date $n=270,146(39.7 \%)$

Include patients with $\mathrm{BMI} \geq 30 \mathrm{~kg} / \mathrm{m}^{2}$ as predicted using the validated machine learning BMI prediction tool using baseline claims $n=127,725(47.3 \%)$

Include patients with $\geq 1$ concurrent medication besides rivaroxaban or warfarin on the index date

$n=121,105(94.8 \%)$

Exclude patients with both rivaroxaban and warfarin pharmacy claims on the index date $\mathrm{n}=121,086(100 \%)$

Exclude patients with $\geq 1$ medical claim with GPI code(s) for any of the oral anticoagulants during the baseline period and on the index date $n=111,897(92.4 \%)$

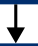

Exclude patients with $\geq 1$ knee or hip replacement code during the baseline period $n=107,700(96.2 \%)$

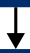

Exclude patients with $\geq 1$ DVT/PE code during the baseline period $\mathrm{n}=101,839(94.6 \%)$

Exclude patients with $\geq 1$ medical claim with ICD-9-CM or ICD-10-CM diagnosis code(s) for mitral stenosis during the baseline period $n=98,171(96.4 \%)$

$\downarrow$

Exclude patients with $\geq 1$ medical claim with HCPCS code(s) for mechanical heart valve procedure during the baseline period $n=97,846(99.7 \%)$

Exclude patients with $\geq 1$ hospitalization or ER visit with a primary diagnosis for stroke/systemic embolism in 30 days prior to the index date $n=96,003(98.1 \%)$

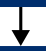

Exclude patients with $\geq 1$ medical claim with any major bleeding events in the 30 days prior to the index date $n=95,875(99.9 \%)$ 
Table 1 Demographic and baseline clinical characteristics

\begin{tabular}{|c|c|c|c|c|c|c|}
\hline \multirow[t]{2}{*}{ Characteristic } & \multicolumn{3}{|c|}{ Before matching } & \multicolumn{3}{|l|}{ After matching } \\
\hline & $\begin{array}{l}\text { Rivaroxaban } \\
{[n=33,191]}\end{array}$ & $\begin{array}{l}\text { Warfarin } \\
{[n=62,684]}\end{array}$ & $\begin{array}{l}\text { Std } \\
\text { difference } \\
(\%)^{\mathrm{a}}\end{array}$ & $\begin{array}{l}\text { Rivaroxaban } \\
{[n=21,547]}\end{array}$ & $\begin{array}{l}\text { Warfarin } \\
{[n=21,547]}\end{array}$ & $\begin{array}{l}\text { Std. } \\
\text { difference } \\
(\%)^{\mathrm{a}}\end{array}$ \\
\hline Age, years [mean $(\mathrm{SD})]$ & $62.97(10.3)$ & $67.72(10.3)$ & 46.0 & $65.14(10.2)$ & $65.28(10.4)$ & 1.3 \\
\hline \multicolumn{7}{|l|}{ Sex } \\
\hline Male & $21,872(65.9)$ & $39,110(62.4)$ & 7.3 & $13,801(64.1)$ & $13,772(63.9)$ & 0.3 \\
\hline Female & $11,319(34.1)$ & $23,574(37.6)$ & 7.3 & $7746(36.0)$ & $7775(36.1)$ & 0.3 \\
\hline \multicolumn{7}{|l|}{ Health insurance type } \\
\hline CCAE & $21,089(63.5)$ & $26,326(42.0)$ & 44.2 & $11,515(53.4)$ & $11,442(53.1)$ & 0.7 \\
\hline MDCR & $12,102(36.5)$ & $36,358(58.0)$ & 44.2 & $10,032(46.6)$ & $10,105(46.9)$ & 0.7 \\
\hline \multicolumn{7}{|l|}{ Baseline clinical characteristics } \\
\hline QCI score [mean (SD)] & $1.51(1.94)$ & $2.03(2.12)$ & 26.0 & $1.75(2.07)$ & $1.84(2.06)$ & 4.1 \\
\hline $\begin{array}{l}\mathrm{CHA}_{2} \mathrm{DS}_{2} \text {-VASc score } \\
{[\text { mean }(\mathrm{SD})]}\end{array}$ & $2.64(1.79)$ & $3.42(1.91)$ & 0.4 & $3.00(1.88)$ & $3.07(1.84)$ & 4.1 \\
\hline HAS-BLED score [mean (SD)] & $2.18(1.37)$ & $2.44(1.47)$ & 0.2 & $2.33(1.43)$ & $2.38(1.46)$ & 3.2 \\
\hline \multicolumn{7}{|l|}{ BMI category } \\
\hline $30.0-34.9 \mathrm{~kg} / \mathrm{m}^{2}$ & $16,340(49.2)$ & $32,865(52.4)$ & 6.4 & $10,926(50.7)$ & $10,909(50.7)$ & 0.2 \\
\hline $35.0-39.9 \mathrm{~kg} / \mathrm{m}^{2}$ & $5156(15.5)$ & $9378(15.0)$ & 1.6 & $3169(14.7)$ & $3159(14.7)$ & 0.1 \\
\hline$\geq 40.0 \mathrm{~kg} / \mathrm{m}^{2}$ & $11,695(35.2)$ & $20,441(32.6)$ & 5.5 & $7452(34.6)$ & $7479(34.7)$ & 0.3 \\
\hline \multicolumn{7}{|l|}{ Common comorbid conditions ${ }^{b}$} \\
\hline Hypertension & $28,370(85.5)$ & $51,146(81.6)$ & 10.5 & $18,417(85.5)$ & $18,047(83.8)$ & 4.8 \\
\hline Hyperlipidemia & $21,418(64.5)$ & $37,960(60.6)$ & 8.2 & $13,922(64.6)$ & $13,425(62.3)$ & 4.8 \\
\hline Mild diabetes & $13,517(40.7)$ & $31,778(50.7)$ & 20.1 & $9703(45.0)$ & $10,008(46.4)$ & 2.8 \\
\hline Osteoarthritis & $8303(25.0)$ & $16,382(26.1)$ & 2.6 & $5726(26.6)$ & $5472(25.4)$ & 2.7 \\
\hline Chronic pulmonary disease & $8240(24.8)$ & $18,839(30.1)$ & 11.7 & $5982(27.8)$ & $6117(28.4)$ & 1.4 \\
\hline Cancer & 7865 (23.7) & $19,306(30.8)$ & 16.0 & $6246(29.0)$ & $6195(28.8)$ & 0.5 \\
\hline Congestive heart failure & $7410(22.3)$ & $21,613(34.5)$ & 27.2 & $5849(27.1)$ & $6407(29.7)$ & 5.7 \\
\hline Thyroid disease & $6395(19.3)$ & $11,691(18.7)$ & 1.6 & $4353(20.2)$ & $4013(18.6)$ & 4.0 \\
\hline Coronary artery disease & $5366(16.2)$ & $10,179(16.2)$ & 0.2 & $3493(16.2)$ & $4010(18.6)$ & 6.3 \\
\hline COPD & $4488(13.5)$ & $12,173(19.4)$ & 16.0 & $3524(16.4)$ & $3774(17.5)$ & 3.1 \\
\hline Chronic diabetes & $4371(13.2)$ & $12,440(19.8)$ & 18.1 & $3412(15.8)$ & $3622(16.8)$ & 2.6 \\
\hline Anemia & $4225(12.7)$ & $12,564(20.0)$ & 19.9 & $4210(19.5)$ & $4319(20.0)$ & 1.3 \\
\hline Cerebrovascular disease & 3962 (11.9) & $11,350(18.1)$ & 17.3 & $3175(14.7)$ & $3428(15.9)$ & 3.3 \\
\hline Chronic kidney disease & $3224(9.7)$ & $12,277(19.6)$ & 28.2 & $2845(13.2)$ & $3255(15.1)$ & 5.5 \\
\hline Peripheral vascular disease & $4134(12.5)$ & $11,265(18.0)$ & 15.4 & $3268(15.2)$ & $3460(16.1)$ & 2.5 \\
\hline \multicolumn{7}{|l|}{ Baseline concomitant drug use } \\
\hline Non-oral anticoagulant & $3735(11.3)$ & $7974(12.7)$ & 4.5 & $2469(11.5)$ & $2611(12.1)$ & 2.0 \\
\hline Antihyperlipidemics & $2478(7.5)$ & $7721(12.3)$ & 16.3 & $1966(9.1)$ & $2187(10.1)$ & 3.5 \\
\hline Antihypertensives & $30,259(91.2)$ & $59,387(94.7)$ & 14.0 & $19,859(92.2)$ & $19,921(92.5)$ & 1.1 \\
\hline Antiplatelet agents & $3640(11.0)$ & $6480(10.3)$ & 2.0 & $2611(12.1)$ & 2699 (12.5) & 1.2 \\
\hline \multicolumn{7}{|l|}{$\begin{array}{l}\text { All-cause HRU, counts } \\
\text { [mean (SD)] }\end{array}$} \\
\hline Inpatient hospitalization & $1.0(1.7)$ & $1.4(2.9)$ & 16.0 & $1.1(2.0)$ & $1.2(2.2)$ & 3.9 \\
\hline ER visit & $0.4(0.9)$ & $0.4(0.9)$ & 4.0 & $0.4(0.9)$ & $0.4(0.9)$ & 0.8 \\
\hline Office visit & $10.5(8.4)$ & $14.3(11.8)$ & 37.0 & $11.4(9.2)$ & $11.9(9.1)$ & 5.2 \\
\hline Outpatient visit & $51.7(54.6)$ & $70.9(92.2)$ & 25.0 & $55.8(60.7)$ & $59.2(63.4)$ & 5.6 \\
\hline Pharmacy fill & $37.4(28.2)$ & $49.6(32.4)$ & 40.0 & $40.6(30.0)$ & $42.1(28.7)$ & 5.3 \\
\hline $\mathrm{SNF} /$ long-term care & 1279 (3.9) & $4002(6.4)$ & 11.5 & $1024(4.8)$ & $1116(5.2)$ & 2.0 \\
\hline
\end{tabular}


Table 1 (continued)

\begin{tabular}{|c|c|c|c|c|c|c|}
\hline \multirow[t]{2}{*}{ Characteristic } & \multicolumn{3}{|l|}{ Before matching } & \multicolumn{3}{|l|}{ After matching } \\
\hline & $\begin{array}{l}\text { Rivaroxaban } \\
{[n=33,191]}\end{array}$ & $\begin{array}{l}\text { Warfarin } \\
{[n=62,684]}\end{array}$ & $\begin{array}{l}\text { Std } \\
\text { difference } \\
(\%)^{\mathrm{a}}\end{array}$ & $\begin{array}{l}\text { Rivaroxaban } \\
{[n=21,547]}\end{array}$ & $\begin{array}{l}\text { Warfarin } \\
{[n=21,547]}\end{array}$ & $\begin{array}{l}\text { Std. } \\
\text { difference } \\
(\%)^{\mathrm{a}}\end{array}$ \\
\hline \multicolumn{7}{|c|}{$\begin{array}{l}\text { All-cause baseline costs, US\$ } \\
\text { [mean (SD)] }\end{array}$} \\
\hline Inpatient cost & $18,234.6(50,446.7)$ & $25,723.8(72,441.7)$ & 12 & $22,805.5(57,615.0)$ & $28,469.8(75,651.6)$ & 8.4 \\
\hline ER cost & $703.3(2393.3)$ & $608.0(2290.5)$ & 4 & $663.6(2266.2)$ & $644.3(2511.5)$ & 0.8 \\
\hline Office visit cost & $1286.6(1207.8)$ & 1557.4 (2081.6) & 16 & $1373.7(1267.0)$ & $1403.9(1600.3)$ & 2.1 \\
\hline Outpatient cost & $10,454.6(24,923.7)$ & $13,112.3(47,570.4)$ & 7 & $10,778.4(26,091.9)$ & $12,364.2(42,685.9)$ & 4.5 \\
\hline Pharmacy cost & $5051.9(12,120.3)$ & $5661.9(11,293.0)$ & 5 & $5159.1(10,664.6)$ & $5649.7(13,182.6)$ & 4.1 \\
\hline SNF cost & $122.3(1496.0)$ & 330.9 (2599.6) & 10 & 179.3 (1839.7) & 226.4 (2275.9) & 2.3 \\
\hline
\end{tabular}

Data are expressed as $n(\%)$ unless otherwise specified

$B M I$ body mass index, $C C A E$ IBM MarketScan Commercial Claims and Encounters database, COPD chronic obstructive pulmonary disease, $E R$ emergency room, $H R U$ healthcare resource utilization, $M D C R$ IBM MarketScan Medicare Supplemental database, $Q C I$ Quan-Charlson comorbidity index, $S t d$ standardized, $S D$ standard deviation, $S N F$ skilled nursing facility

${ }^{\text {a }}$ Std difference $<10 \%$ was considered a negligible imbalance

${ }^{b} \geq 15 \%$ in any treatment cohort before matching
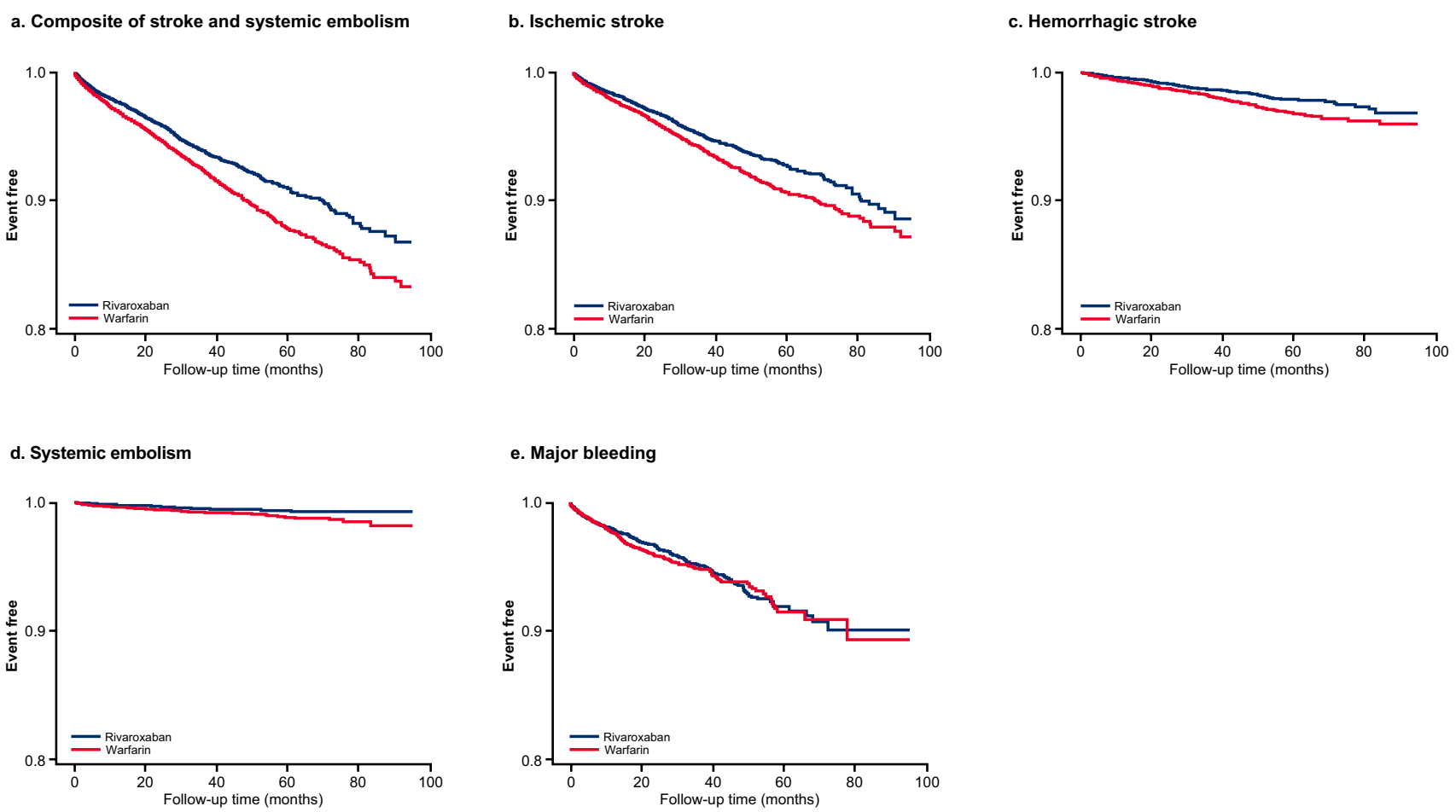

Fig. 3 Kaplan-Meier curves of clinical outcomes with rivaroxaban versus warfarin in the overall population

cohorts, respectively $(p<0.0001)$. The mean (SD) PDC at discontinuation was $0.94(0.09)$ in the rivaroxaban cohort versus $0.92(0.11)$ in the warfarin cohort. The mean PDC during the entire follow-up period was $0.61(0.36)$ versus $0.60(0.33)$ in the rivaroxaban versus warfarin cohorts, respectively. At the time of index treatment discontinuation, more rivaroxaban patients achieved $\geq 80 \%$ of days covered versus warfarin patients $(92.4 \%$ vs. $86.2 \% ; p<0.0001)$. Throughout the entire follow-up period, a higher proportion of patients receiving rivaroxaban achieved $\geq 80 \%$ of days covered versus those receiving warfarin $(46.6 \%$ vs. $38.1 \%$; $p<0.0001)$. 


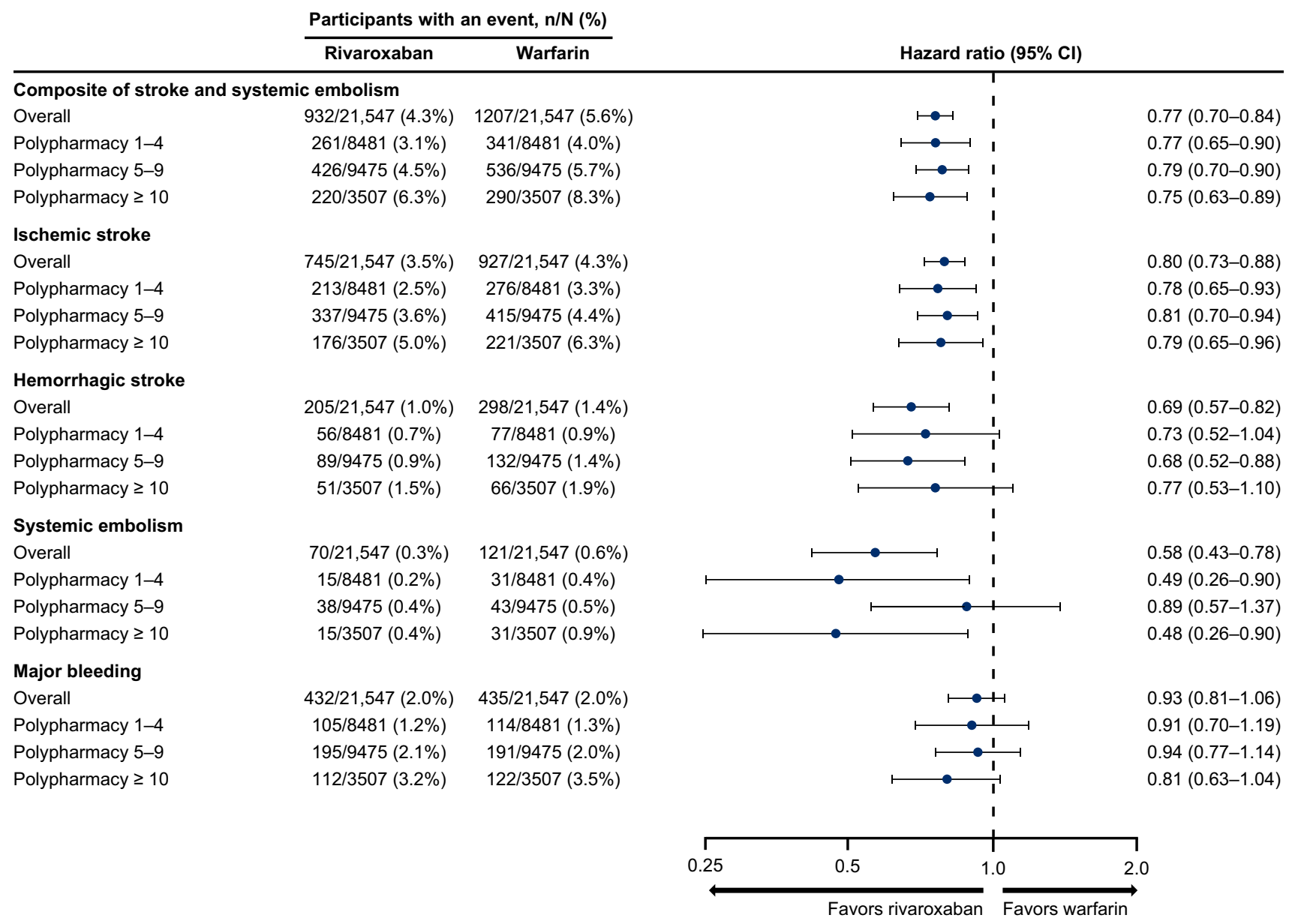

Fig. 4 Risk of clinical outcomes with rivaroxaban and warfarin overall and by polypharmacy subgroups. $C I$ confidence interval

\section{Discussion}

The results of this real-world analysis showed that among NVAF patients with obesity and polypharmacy, those receiving rivaroxaban had a lower risk of stroke and systemic embolism compared with warfarin overall and regardless of polypharmacy or BMI category. Lower rates of ischemic stroke, hemorrhagic stroke, and systemic embolism were observed with rivaroxaban versus warfarin overall and in several of the polypharmacy and BMI subgroups; however, statistical significance was not achieved in all polypharmacy and BMI subgroups due to sample size. Numerical trends were generally noted in all groups with similar HRs, but statistical significance was achieved in the subgroups with larger sample sizes or where a larger number of events occurred. The risk of major bleeding was not significantly different between rivaroxaban and warfarin overall or across polypharmacy categories. Persistence and adherence to treatment with rivaroxaban were higher than those of warfarin. The current analysis did not evaluate drug-drug interactions between the anticoagulants and other drugs. Drug-drug interactions are not captured in claims data and would only be identified from clinician notes.

These findings are consistent with previous analyses of clinical trial and real-world data. The ROCKET-AF trial population had moderate-to-high polypharmacy (5-9 medications), which was similar in our study (64\% vs. $60 \%$ ) [16]. In a subgroup analysis of the ROCKET-AF trial, Piccini et al. examined the risk of stroke and bleeding events between rivaroxaban and warfarin according to the number of concurrent medications (0-4, 5-9, and $\geq 10)$. Across all polypharmacy groups, stroke or non-CNS embolism events were numerically higher in the warfarin group, whereas major bleeding was similar and intracranial hemorrhage was less frequent in the rivaroxaban versus warfarin group. A separate post hoc analysis of the ROCKET-AF trial found lower rates of stroke and systemic embolism in overweight and obese patients compared with normal weight patients with both rivaroxaban and warfarin, and bleeding rates were similar across all weight groups [36].

Real-world evidence studies have demonstrated the effectiveness and safety of rivaroxaban in NVAF patients with 


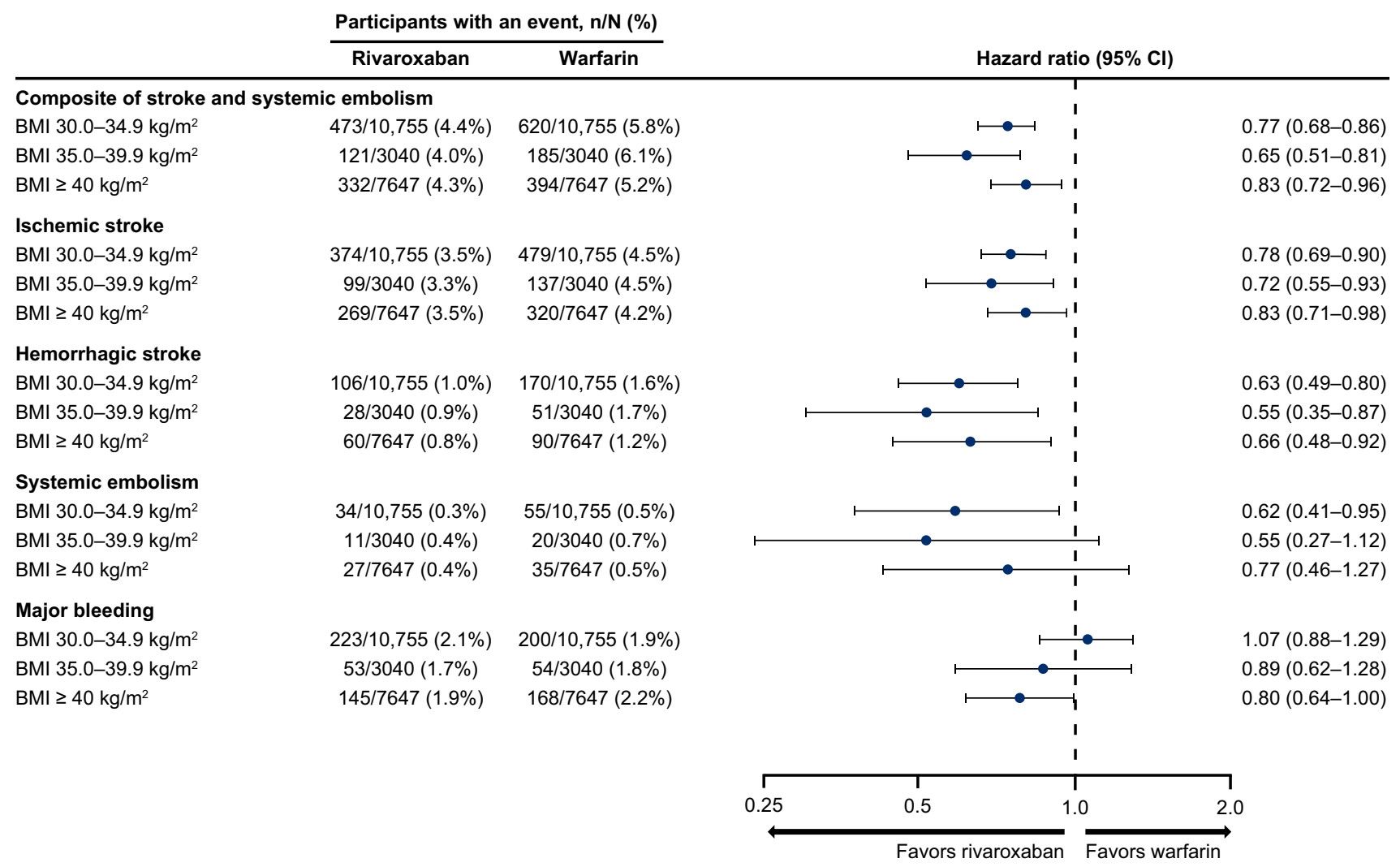

Fig. 5 Risk of clinical outcomes with rivaroxaban and warfarin in the BMI subgroups. BMI body mass index, $C I$ confidence interval

obesity [23-27]. Using two US healthcare claims databases, the composite risk of stroke and systemic embolism and the risk of major bleeding were not significantly different between rivaroxaban and warfarin in patients with morbid obesity [23]. Electronic health record data for NVAF patients with obesity found a reduced risk of stroke and systemic embolism as well as major bleeding for rivaroxaban versus warfarin, with no significant interaction across BMI categories of 30 to $<35 \mathrm{~kg} / \mathrm{m}^{2}, \geq 35$ to $<40 \mathrm{~kg} / \mathrm{m}^{2}$, and $\geq 40$ $\mathrm{kg} / \mathrm{m}^{2}$ [25]. In a retrospective cohort study of patients with $\mathrm{BMI}>40 \mathrm{~kg} / \mathrm{m}^{2}$ or weight $>120 \mathrm{~kg}$ from two academic medical centers, the composite endpoint of clinical failure of anticoagulation (venous thromboembolism recurrence, stroke incidence, or mortality) trended lower with rivaroxaban versus warfarin but was not statistically significant $(5 \%$ vs. $13 \% ; p=0.06$ ) [24]. A single-center, retrospective chart review found similar efficacy and safety for rivaroxaban and warfarin among morbidly obese patients (BMI $\geq 40 \mathrm{~kg} / \mathrm{m}^{2}$ ) [26]. Lastly, a US commercial claims retrospective analysis found $26 \%$ lower risk of stroke and systemic embolism among NVAF obese patients prescribed rivaroxaban, with similar major bleeding compared with warfarin at 36 months follow-up [27].

Separate real-world evidence studies have also evaluated the effectiveness and safety of rivaroxaban in NVAF patients with polypharmacy [28, 29]. An analysis of Medicare patients with low, moderate, or high polypharmacy (i.e., $\leq 3,4-8, \geq 9$ other prescription medications, respectively) found similar risk of ischemic stroke, bleeding, and death with rivaroxaban compared with warfarin [28]. Another retrospective study by Martinez et al. [29] evaluated the safety and effectiveness of rivaroxaban and warfarin among polypharmacy users ( $\geq 5$ or $\geq 10$ medications). In the $\geq 5$ medication analysis, rivaroxaban was associated with a lower risk of stroke/systemic embolism and ischemic stroke alone with similar major bleeding compared with warfarin, whereas no significant differences were found in the $\geq 10$ medications analysis, likely due to being underpowered given the smaller sample size [29].

Approximately $29 \%$ of patients in this analysis had a cancer diagnosis at baseline, which may or may not have been active disease. This proportion of patients with cancer is consistent with the proportion of patients with AF and a history of cancer in the ORBIT-AF registry (23.8\%) [37]. Patients in the ORBIT-AF registry who had cancer carried a higher burden of cardiovascular disease risk factors and disease but were similarly treated with antithrombotics and antiplatelet therapies as their counterparts without cancer. No differences were observed in the risks of strokes/nonCNS embolism, cardiovascular death, and heart failure 
between AF patients with and without a history of cancer, although the former were characterized by a higher risk of major bleeding and non-cardiovascular death. We did not define the cancer population as a separate subgroup for this analysis because they may not have been active diagnoses and requiring two active diagnoses would have reduced the sample size. Chronic kidney disease (CKD) was identified in $14 \%$ of patients based on diagnostic codes. Laboratory data were not available to determine glomerular filtration rate. Similar to the cancer population, we did not evaluate particular subpopulations beyond those with obesity and polypharmacy. However, in the ORBIT-AF registry, 39\% of patients had an estimated creatinine clearance of $<60 \mathrm{~mL} / \mathrm{min}$ and these patients received fewer arrhythmia treatments, while those with advanced CKD were less likely to receive oral anticoagulants [38]. Our study may have had a lower proportion of patients with CKD compared with the registry because we focused on the obese population, which tended to be younger and have higher median creatinine clearance versus normal weight and overweight patients in the registry [39].

Our geographically diverse claims database analysis provided results that are generalizable to both commercially insured and Medicare supplemental beneficiaries across the US. Using 12 months of continuous health plan enrollment allowed better understanding of patient characteristics and longitudinal evaluation of outcomes, and selection biases were reduced by using PSM techniques.

Our study has several limitations worth mentioning. This analysis was limited to the defined subgroups by BMI and polypharmacy. Further subdivisions of these subgroups would have reduced sample sizes and decreased the power to identify significant findings. The use of administrative claims data is subject to potential coding errors and inconsistencies. BMI was not available directly from the claims data and was therefore identified using a validated machine learning algorithm, which may misclassify patients. However, a sensitivity analysis using diagnosis codes for obesity showed consistent results with those obtained using the BMI algorithm. Time in the therapeutic international normalized ratio range for warfarin patients was not assessed due to a lack of complete laboratory data in these claims databases. Results are limited to the patient population included in this study and may not be generalizable to the broader populations (e.g., Medicaid, uninsured, other countries). Prescription claims do not indicate that the medication was taken as prescribed, and over-the-counter medication use and medication samples may not be captured in claims data. In addition, there was no assessment of adherence to the baseline medications such as antiplatelets and non-oral anticoagulants. Residual confounding cannot be excluded even with PSM due to potential unmeasured confounders.

\section{Conclusion}

This study of real-world patients demonstrated that NVAF patients with obesity and polypharmacy receiving rivaroxaban had a significantly lower risk of stroke and systemic embolism compared with those receiving warfarin, with similar risks of major bleeding between cohorts in all polypharmacy categories. Patients receiving rivaroxaban had a significantly higher rate of treatment persistence and adherence, supporting the use of rivaroxaban as an alternative to warfarin in this population.

Supplementary Information The online version contains supplementary material available at https://doi.org/10.1007/s40256-021-00520-7.

Acknowledgements Medical writing support was provided by Leo J. Philip Tharappel (SIRO Clinpharm Pvt Ltd) and Oghenetega Umukoro, $\mathrm{PhD}$, of Cello Health Communications/MedErgy (Yardley, PA, USA), which was funded by Janssen Scientific Affairs, LLC (Titusville, NJ, USA).

\section{Declarations}

Funding This study was funded by Janssen Scientific Affairs, LLC (Titusville, NJ, USA).

Competing interests Mark J. Alberts has not received compensation for this project but received consultancy fees from Janssen Pharmaceuticals and Janssen Scientific Affairs for being on the Steering Committee of the Quantum AF study. Jinghua He, Akshay Kharat, and Veronica Ashton are full-time employees of Janssen Scientific Services, LLC.

Availability of data and material Not applicable.

Code availability Not applicable.

Ethics approval and consent to participate Not applicable.

Consent for publication Not applicable.

Author contributions All authors participated in the design and analytical approach of the study and contributed to the manuscript development. Results were summarized and interpreted in collaboration with all authors. The authors vouch for the accuracy and completeness of the data reported and the adherence of the study to the protocol. All authors gave final approval of the version to be published.

Open Access This article is licensed under a Creative Commons Attribution-NonCommercial 4.0 International License, which permits any non-commercial use, sharing, adaptation, distribution and reproduction in any medium or format, as long as you give appropriate credit to the original author(s) and the source, provide a link to the Creative Commons licence, and indicate if changes were made. The images or other third party material in this article are included in the article's Creative Commons licence, unless indicated otherwise in a credit line to the material. If material is not included in the article's Creative Commons licence and your intended use is not permitted by statutory regulation or exceeds the permitted use, you will need to obtain permission directly from the copyright holder. To view a copy of this licence, visit http://creativecommons.org/licenses/by-nc/4.0/. 


\section{References}

1. January CT, Wann LS, Alpert JS, Calkins H, Cigarroa JE, Cleveland JC Jr, et al. 2014 AHA/ACC/HRS guideline for the management of patients with atrial fibrillation: a report of the American College of Cardiology/American Heart Association Task Force on practice guidelines and the Heart Rhythm Society. Circulation. 2014;130:e199-267.

2. Hindricks G, Potpara T, Dagres N, Arbelo E, Bax JJ, BlomströmLundqvist C, et al. 2020 ESC guidelines for the diagnosis and management of atrial fibrillation developed in collaboration with the European Association for Cardio-Thoracic Surgery (EACTS). The task force for the diagnosis and management of atrial fibrillation of the European Society of Cardiology (ESC). Developed with the special contribution of the European Heart Rhythm Association (EHRA) of the ESC. Eur Heart J. 2021;42:373-498.

3. Adair T, Lopez AD. The role of overweight and obesity in adverse cardiovascular disease mortality trends: an analysis of multiple cause of death data from Australia and the USA. BMC Med. 2020;18:199.

4. Raebel MA, Malone DC, Conner DA, Xu S, Porter JA, Lanty FA. Health services use and health care costs of obese and nonobese individuals. Arch Intern Med. 2004;164:2135-40.

5. Edwards CH, Aas E, Kinge JM. Body mass index and lifetime healthcare utilization. BMC Health Serv Res. 2019;19:696.

6. Hales CM, Carroll MD, Fryar CD, Ogden CL. Prevalence of obesity and severe obesity among adults: United States, 2017-2018. NCHS Data Brief. 2020;360:1-8.

7. OECD. Obesity update 2017. https://www.oecd.org/health/healthsystems/Obesity-Update-2017.pdf. Accessed 10 Dec 2021.

8. Finkelstein EA, Khavjou OA, Thompson H, Trogdon JG, Pan L, Sherry B, et al. Obesity and severe obesity forecasts through 2030 . Am J Prev Med. 2012;42:563-70.

9. Wang TJ, Parise H, Levy D, D'Agostino RB Sr, Wolf PA, Vasan RS, et al. Obesity and the risk of new-onset atrial fibrillation. JAMA. 2004:292:2471-7.

10. Wong CX, Sullivan T, Sun MT, Mahajan R, Pathak RK, Middeldorp $\mathrm{M}$, et al. Obesity and the risk of incident, post-operative, and post-ablation atrial fibrillation: a meta-analysis of 626,603 individuals in 51 studies. JACC Clin Electrophysiol. 2015;1:139-52.

11. Pouwels S, Topal B, Knook MT, Celik A, Sundbom M, Ribeiro R, et al. Interaction of obesity and atrial fibrillation: an overview of pathophysiology and clinical management. Expert Rev Cardiovasc Ther. 2019;17:209-23.

12. Martin K, Beyer-Westendorf J, Davidson BL, Huisman MV, Sandset PM, Moll S. Use of the direct oral anticoagulants in obese patients: guidance from the SSC of the ISTH. J Thromb Haemost. 2016;14:1308-13.

13. Nalliah CJ, Sanders P, Kottkamp H, Kalman JM. The role of obesity in atrial fibrillation. Eur Heart J. 2016;37:1565-72.

14. Kit BK, Ogden CL, Flegal KM. Prescription medication use among normal weight, overweight, and obese adults, United States, 2005-2008. Ann Epidemiol. 2012;22:112-9.

15. Guh DP, Zhang W, Bansback N, Amarsi Z, Birmingham CL, Anis $\mathrm{AH}$. The incidence of co-morbidities related to obesity and overweight: a systematic review and meta-analysis. BMC Public Health. 2009;9:88.

16. Piccini JP, Hellkamp AS, Washam JB, Becker RC, Breithardt G, Berkowitz SD, et al. Polypharmacy and the efficacy and safety of rivaroxaban versus warfarin in the prevention of stroke in patients with nonvalvular atrial fibrillation. Circulation. 2016;133:352-60.

17. Coumadin (warfarin) [package insert]. Princeton, NJ: BristolMyers Squibb Company; 2011.
18. January CT, Wann LS, Calkins H, Chen LY, Cigarroa JE, Cleveland JC Jr, et al. 2019 AHA/ACC/HRS focused update of the 2014 AHA/ACC/HRS guideline for the management of patients with atrial fibrillation. Circulation. 2019;140:e125-51.

19. Sebaaly J, Kelley D. Direct oral anticoagulants in obesity: an updated literature review. Ann Pharmacother. 2020;54:1144-58.

20. Grymonprez M, De Backer TL, Steurbaut S, Boussery K, Lahousse L. Non-vitamin $\mathrm{k}$ antagonist oral anticoagulants (NOACs) versus warfarin in patients with atrial fibrillation and (morbid) obesity or low body weight: a systematic review and meta-analysis. Cardiovasc Drugs Ther. 2021. https://doi.org/10. 1007/s10557-020-07122-6 (Epub 11 Jan 2021).

21. Patel MR, Mahaffey KW, Garg J, Pan G, Singer DE, Hacke W, et al. Rivaroxaban versus warfarin in nonvalvular atrial fibrillation. N Engl J Med. 2011;365:883-91.

22. Xarelto ${ }^{\circledR}$ (rivaroxaban) tablets, for oral use [prescribing information]. Titusville, NJ: Janssen Pharmaceuticals, Inc.; 2021.

23. Peterson ED, Ashton V, Chen YW, Wu B, Spyropoulos AC. Comparative effectiveness, safety, and costs of rivaroxaban and warfarin among morbidly obese patients with atrial fibrillation. Am Heart J. 2019;212:113-9.

24. Perales IJ, San Agustin K, DeAngelo J, Campbell AM. Rivaroxaban versus warfarin for stroke prevention and venous thromboembolism treatment in extreme obesity and high body weight. Ann Pharmacother. 2020;54:344-50.

25. Costa OS, Beyer-Westendorf J, Ashton V, Milentijevic D, Moore KT, Bunz TJ, et al. Effectiveness and safety of rivaroxaban versus warfarin in obese nonvalvular atrial fibrillation patients: analysis of electronic health record data. Curr Med Res Opin. 2020;36:1081-8.

26. Kushnir M, Choi Y, Eisenberg R, Rao D, Tolu S, Gao J, et al. Efficacy and safety of direct oral factor Xa inhibitors compared with warfarin in patients with morbid obesity: a single-centre, retrospective analysis of chart data. Lancet Haematol. 2019;6:e359-65.

27. Berger JS, Laliberté F, Kharat A, Lejeune D, Moore KT, Jung Y, et al. Real-world effectiveness and safety of rivaroxaban versus warfarin among non-valvular atrial fibrillation patients with obesity in a US population. Curr Med Res Opin. 2021;37:881-90.

28. Mentias A, Heller E, Vaughan SM. Comparative effectiveness of rivaroxaban, apixaban, and warfarin in atrial fibrillation patients with polypharmacy. Stroke. 2020;51:2076-86.

29. Martinez BK, Baker WL, Sood NA, Bunz TJ, Meinecke AK, Eriksson D, et al. Influence of polypharmacy on the effectiveness and safety of rivaroxaban versus warfarin in patients with nonvalvular atrial fibrillation. Pharmacotherapy. 2019;39:196-203.

30. Wu B, Chow W, Sakthivel M, Kakade O, Gupta K, Israel D, et al. Body mass index variable interpolation to expand the utility of real-world administrative healthcare claims database analyses. Adv Ther. 2021;38:1314-27.

31. Cunningham A, Stein CM, Chung CP, Daugherty JR, Smalley WE, Ray WA. An automated database case definition for serious bleeding related to oral anticoagulant use. Pharmacoepidemiol Drug Saf. 2011;20:560-6.

32. Quan H, Li B, Couris CM, Fushimi K, Graham P, Hider P, et al. Updating and validating the Charlson comorbidity index and score for risk adjustment in hospital discharge abstracts using data from 6 countries. Am J Epidemiol. 2011;173:676-82.

33. Lip GY, Nieuwlaat R, Pisters R, Lane DA, Crijns HJ. Refining clinical risk stratification for predicting stroke and thromboembolism in atrial fibrillation using a novel risk factor-based approach: the Euro Heart Survey on atrial fibrillation. Chest. 2010;137:263-72. 
34. Pisters R, Lane DA, Nieuwlaat R, de Vos CB, Crijns HJ, Lip GY. A novel user-friendly score (HAS-BLED) to assess 1-year risk of major bleeding in patients with atrial fibrillation: the Euro Heart Survey. Chest. 2010;138:1093-100.

35. Austin PC. The use of propensity score methods with survival or time-to-event outcomes: reporting measures of effect similar to those used in randomized experiments. Stat Med. 2014;33:1242-58.

36. Balla SR, Cyr DD, Lokhnygina Y, Becker RC, Berkowitz SD, Breithardt $\mathrm{G}$, et al. Relation of risk of stroke in patients with atrial fibrillation to body mass index (from patients treated with rivaroxaban and warfarin in the rivaroxaban once daily oral direct factor Xa inhibition compared with vitamin $\mathrm{K}$ antagonism for prevention of stroke and embolism trial in atrial fibrillation trial). Am J Cardiol. 2017;119:1989-96.
37. Melloni C, Shrader P, Carver J, Piccini JP, Thomas L, Fonarow GC, et al. Management and outcomes of patients with atrial fibrillation and a history of cancer: the ORBIT-AF registry. Eur Heart J Qual Care Clin Outcomes. 2017;3:192-7.

38. Washam JB, Holmes DN, Thomas LE, Pokorney SD, Hylek EM, Fonarow GC, et al. Pharmacotherapy for atrial fibrillation in patients with chronic kidney disease: insights from ORBIT-AF. J Am Heart Assoc. 2018;7:e008928.

39. Pandey A, Gersh BJ, McGuire DK, Shrader P, Thomas L, Kowey $\mathrm{PR}$, et al. Association of body mass index with care and outcomes in patients with atrial fibrillation: results from the ORBIT-AF Registry. JACC Clin Electrophysiol. 2016;2:355-63. 